\title{
Anti-Viral Vaccine Activity of Zinc(II) for Viral Prevention, Entry, Replication, and Spreading During Pathogenesis Process
}

\author{
Tsuneo Ishida* \\ Doctor of Science, Tohoku University, Japan
}

Submission: May 23, 2019; Published: July 09, 2019

*Corresponding author: Dr. Sci. Tsuneo Ishida, Prof of Teikyo University, and Prof of Yamazaki Gakuen University, Retirement, 2-3-6, Saido, Midori-Ku, Saitama-Shi, Saitama-Ken, T336-0907, Japan

\begin{abstract}
Anti-viral vaccine activity of $\mathrm{Zn}^{2+}$ ions for viral prevention, pathogenesis processes, and ROS generation causing to oxidative stress have been investigated. AZP is efficient for viral prevention by inhibitions of BSCTV and DNA virus replications. The AZP phenotypes show strongly resistant to virus infection and viral DNA replication could be applied to the prevention of virus infections in humans. ZnOTs exhibit the ability to neutralize HSV-2 virions and blocking HSV-2 attachment activity. Zinc salts can mediate antiviral activity on RSV by altering the ability of the cell to support RSV replication. The effect of zinc sulfate on seroconversion after a simple method vaccination had been identified that accelerated HB vaccination can shorten duration of immunization of this clinical trial for showing its effectiveness. The inhibition of zinc binding activity of hMPV M2-1 protein can lead to the development of novel, live attenuated vaccines as well as antiviral drugs for pneumoviruses. The CCHH zinc finger motif provides potential vaccine candidates for the development of live species-specific attenuated influenza virus vaccines. Chelates zinc ions inhibit HIV-1 replication. The LAIVs are attracting attention as several advantages over inactivated vaccines. Zinc finger reactive compounds also inactivate retroviruses. ZOTEN promoted the presentation of bound HSV-2 virions.
\end{abstract}

The regulated ZFNs in the presence of HIV-1 Tat may provide a safer and novel genome-editing technology for eradicating HIV-1 proviral DNA from infected host cells. Zinc ions inhibit vaccine virus growth. The ZAP inhibits HIV-1 infection by viral mRNAs degradation. ZAP also inhibits the spreading against XMRV. The treatment of viral infections with viral spreading will be achieved and improved the therapeutic effects by using zinc oxide nanoparticles. ROS and RNS within viruses of viral entry, viral replication, and viral spread are generated in all situations. ROS in virus pathogenesis play an important role in cell signaling and regulate hormone action, growth factors, cytokines, transcription, apoptosis, immunomodulation, and neuromodulation, leading to chronic oxidative stress. Oxidative stress has been occurred in various viral infections. The antioxidant components lead to an excess storage of $\mathrm{H}_{2} \mathrm{O}_{2}$, which further increases the hydroxyl radicals and lipid peroxide that signal the cell to undergo a programmed cell death. Thus, zinc-associated vaccine activity mechanism against viruses is indicated that the anti-viral vaccine activity of released $\mathrm{Zn}^{2+}$ ions from zinc solutions and $\mathrm{ZnO} \mathrm{NPs}$, may be enhanced by $\mathrm{Zn}^{2+}$ ion-induced $\mathrm{Zn}^{2+}$ ions-coordinated adapted immunity, viral growth regulation, and viral apoptosis and death.

Keywords: AZP/ZOTEN/ZAP/ZFN; Zinc salts and zinc sulfate; ROS; Oxidative stress; HIV/hMPV/ LAV/RSV/XMRV

Abbreviations: APCs: Antigen Presenting Cell; AZP: Artificial Zinc Finger Protein; BSCTV: Beet Severe Curly Top Virus; HIV: Human Immunodeficiency Virus; hMPV: Human Metapneumovirus; HBV: Hepatitis B Virus; HEV: Hepatitis E Virus; HSV2: Herpes Simplex Virus Type2; IRF3: Interferon Regulatory Factor 3; ISREs: Interferon Stimulated Response Elements; LAIVs: Live Attenuated Influenza Vaccines; LAV: Live Attenuated Virus; NKT: Natural Killer T; PLZF: Promyelocytic Leukemia Zinc Finger; RdRp: RNA-Dependent RNA Polymerase; RNS: Reactive Nitrogen Species; ROS: Reactive Oxygen Species; RSV: Respiratory Syncytial Virus, SSP: Stable Signal Peptide; TALENs: Transcription ActivatorLike Effector Nucleases; XMRV: Xenotropic Murine Leukemia Virus related Virus; ZBD: Zinc-Binding Domain; ZAP: Zinc-Finger Antiviral Protein; ZFN: Zinc Finger Nuclease; ZnOTs: ZINC OXIDE TETRAPODS; ZOTENs: Zinc Oxide Tetrapod Nanoparticles

\section{Introduction}

Viruses are small protein capsid that harbor genetic information. In the case of enveloped viruses, an additional lipid bilayer surrounds the capsid that enveloped viruses can spread via two distinct routes, either through the cell-free aqueous environment or, alternatively, by remaining cell associated and being passed on by direct cell-cell contact [1].
The other, zinc is the second most abundant trace metal with human body $2-3 \mathrm{~g}, 90 \%$ in muscle and bone, and $10 \%$ other organs include prostate, liver, the gastrointestinal tract, kidney, skin, lung brain, heart, and pancreas in humans which cellular zinc underlies an efficient homeostatic control that avoids accumulation of zinc in excess [2]. The role of zinc in cell death 
has apoptosis that the influence of zinc on apoptosis is tissue/ cell type, zinc concentration, and expression of zinc transporters and zinc-binding proteins [2]. Zinc has a rather low toxicity and influences apoptosis by acting on several molecular regulators of programmed cell death, including caspases and proteins from the $\mathrm{Bcl}$ and Bax families.

Zinc induced anti-virus activity may be enhanced for Tcell division, maturation and differentiation, lymphocyte response to mitogens, programmed cell death of lymphoid and myeloid origins, gene transcription, and biomembrane function [3]. Nonstructure NS5A is a zinc metalloprotein and zinc coordination is likely required for NS5A function in the hepatitis $C$ replicase that reduced NS5A zinc coordination and zinc binding motif were tolerated for replication [4]. The zinc-finger antiviral protein (ZAP) induced virus during viral infection little is known, however, mutational analysis of the human ZAP promoter revealed that multiple interferon stimulated response elements (ISREs) distal to the transcription start site serve redundantly to control interferon regulatory factor 3 (IRF3)-dependent induction of ZAP trancription [5]

Anti-virus vaccine principle is to viral prevention and devoid serious diseases that live attenuated virus (LAV) vaccines have provided ideal protection from several major diseases. The ability to create a zinc finger nuclease (ZFN) vaccine that can prevent and eliminate persistent viral infections is a long way from being realized, in which the efficacies by available LAV, live attenuated rotavirus, influenza, and varicella zoster vaccines are strong incentive to redouble efforts to improve the safety characteristics of this type of vaccine [6]. Vaccines for numerous infectious diseases have been developed using whole inactivated virions that live attenuated influenza vaccines (LAIVs) are particularly attracting attention as effective strategy due to several advantages over inactivated vaccines [7].

Zinc-binding activity of human metapneumovirus (M21) protein was found to incorporate zinc ions, although the specific roles of the zinc binding activity in viral replication and pathogenesis remains unclear [8]. The pathogenic process, namely viral pathogenesis is the process by which an infection leads to disease that pathogenic mechanisms of viral disease include viral entry, local replication, and spread to organs and shedding of disease site [9]. Accordingly, the zinc induced Zn2+ ion coordinated activity results in regulation of viral growth and may lead to virus death in host cell-virus interaction during pathogenesis process.

In this review, zinc-mediated anti-viral vaccine activities have been investigated for viral prevention, viral entry, replication/ DNA/RNA virus, and spreading to organs during pathogenesis process. Lastly, ROS productions and leading to oxidative stress and virus death are discussed.

\section{Viral prevention}

Viral prevention is a major objective in human health. One attractive approach to the prevention is inhibition of virus replication. Artificial zinc finger proteins (AZP) have been targeted to the replication origin of the Beet severe curly top virus (BSCTV), a model DNA virus that the AZP efficiently blocked binding of the viral replication protein, which initiates virus replication, to the replication origin. These results showing to the AZP phenotypes strongly resistant to virus infection and viral DNA replication could be applied to the prevention of virus infections in humans [10]. Inhibition of virus DNA replication by AZP is comparable to viral prevention. Zinc oxide tetrapods (ZnOTs) exhibit the ability to neutralize herpes simplex virus type-2 (HSV-2) virions and blocking HSV-2 attachment activity by ZnOTs can have prophylactic as well as therapeutic applications [11].

\section{Viral entry and replication}

Zinc salts was examined in the presence of zinc during preincubation, adsorption, or penetration by the degree of inhibition of respiratory syncytial virus (RSV) replication, the resulting that zinc can mediate antiviral activity on RSV by altering the ability of the cell to support RSV replication [12]. Further, zinc salts did not show any effect on the entry of g-1 hepatitis E virus (HEV) into the host cell, directly inhibit the activity of the viral RNA-dependent RNA polymerase (RdRp), leading to inhibition of viral replication, suggesting their possible therapeutic value in controlling HEV infection [13]. The other, Zinc sulfate has no effect in level of immunity among elderly and zinc may stimulate antibody response in the nonresponders to recombinant hepatitis B vaccine. The effect of zinc sulfate on seroconversion after a simple method vaccination had been identified that accelerated hepatitis B vaccination can shorten duration of immunization of this clinical trial for showing its effectiveness [14]. The inhibition of zinc binding activity of human metapneumovirus (hMPV) M2-1 protein can lead to the development of novel, live attenuated vaccines as well as antiviral drugs for pneumoviruses [8].

The CCHH zinc finger motif provides a critical determinant for virulence in mouse and mutations in the CCHH motif yield potential vaccine candidates for the development of live speciesspecific attenuated influenza virus vaccines [15]. Chelates zinc ions (a bisthiadiazolbenzene-1,2-diamine) from retroviral nucleocapsid zinc fingers inhibit HIV-1 replication [16]. This compound thus acts via a different mechanism than the previously reported zinc ejectors, as its structural features do not allow an acyl transfer to Cys or a thioldisulfide interchange. The cleaved stable signal peptide (SSP) is retained in GPC through interaction with a zincbinding domain (ZBD) in the cytoplasmic tail of G2 [17]. The live attenuated influenza vaccines (LAIVs) are attracting attention as an effective strategy due to several 
advantages over inactivated vaccines [18]. Zinc finger reactive compounds also inactivate retroviruses by targeting the zinc finger motif in their nucleocapsid proteins. These compounds are effective against respiratory syncytial virus (RSV) that AT2-inactivated RSV vaccine may not be enough to produce a highly efficacious inactivated virus vaccine which does not lead to an atypical disease [19]. Intravaginal zinc oxide tetrapod nanoparticles (ZOTEN) promoted the presentation of bound HSV-2 virions to mucosal APCs, enhancing T cell-mediated and $\mathrm{Ab}$-mediated responses to the infection, and thereby suppressing a reinfection [20].

\section{DNA/RNA virus inhibitors}

A human papillomavirus type 16 E7 DNA vaccine indicated a significantly stronger E7-specific cytotoxic T-lymphocyte induction and better antitumor protection [21]. Zinc finger nucleases (ZFNs) can specifically and efficiently excise HIV-1 proviral DNA from latently infected human $\mathrm{T}$ cells, the resulting from HIV-1infected human primary T cells and latently infected $T$ cells treated with the inducible ZFNs validated that proviral DNA can be excised [22]. The regulated ZFNs in the presence of HIV-1 Tat may provide a safer and novel genome-editing technology for eradicating HIV-1 proviral DNA from infected host cells. ZFNs and transcription activator-like effector nucleases (TALENs) bear sequence-specific DNA-binding modules that recognize HIV DNA sequences [23]. Therapeutic potential of ZFNs and zinc finger arrays (ZFAs) is confirmed with unique specificity to HSV-2 the genome which ZFNs with specificity to HSV-2 genomic DNA are precursors of novel host genome expressed HSV-2 gene-therapeutics or vaccines [24]. Zinc supplementation in cultured HeLa cells inhibits DNA laddering \& caspase-3 activity, and inhibitory effect on influenza virus induced apoptotic death can be determined at an early stage of the infection by zinc treatment [25]. Further, zinc ions inhibit vaccine virus growth, and a fraction of RNA which is normally synthesized infected cells, was missing from a proper part of the gradient if the cell were treated with zinc ions within $1 \mathrm{~h}$ post infection that a transcriptional step is involved in zinc-caused inhibition of vaccinia virus growth [26]. The zinc-finger antiviral protein (ZAP) inhibits HIV-1 infection by viral mRNAs degradation that ZAP inhibits HIV-1 by recruiting both the 5' and 3' mRNA degradation to specifically promote the degradation of multiply spliced HIV-1 mRNA [27].

Higher levels of dietary zinc oxide ( $\mathrm{ZnO}$ ) levels could not provide enhanced protection against porcine reproductive and respiratory syndrome virus (PRRSV) vaccine and infection that have the potential to stimulate or modulate systemic immune responses after vaccination [28]. In the synthesized $\mathrm{ZnO}$ tetrapod nanoparticles (ZOTENs), ZOTENs prevent HSV-2 infection and disease that ZOTEN provides a platform for virus capture and presentation of neutralized virions to mucosal antigen presenting cell (APCs), for initiating and boosting adaptive immunity which provides the novel evidence for the protective efficacy of an intravaginal microbicide/vaccine or microbivac platform against primary and secondary female genital herpes infection [29].

\section{Viral spread inhibitor}

ZAP inhibits the spreading of replication-competent xenotropic murine leukemia virus-related virus (XMRV) which ZAP inhibits viral infection [30]. The treatment of viral infections with viral spreading will be achieved and improved the therapeutic effects by using zinc oxide nanoparticles [31].

ROS Generation in Host Cell-Virus Interaction and Leading to Virus Growth Regulation and Virus Death

Reactive oxygen species (ROS) and reactive nitrogen species (RNS) within viruses of viral entry, viral replication, and viral spread are generated in all situations. ROS induce several cellular effects, including cell cycle progression, apoptosis, DNA damage, senescence, and neurodegeneration. ROS in virus pathogenesis play an important role in cell signaling and regulate hormone action, growth factors, cytokines, transcription, apoptosis, immunomodulation, and neuromodulation, leading to chronic oxidative stress [32]. Oxidative stress has been occurred in various viral infections. For human immunodeficiency virus (HIV), the body antioxidant system becomes weaker as HIV progresses that the imbalances inside and outside the cell influence the cell to undergo a programmed cell death [32]. The antioxidant components lead to an excess storage of H2O2, which further increases the hydroxyl radicals and lipid peroxide that signal the cell to undergo a programmed cell death [32]. The influenza virus induces ROS production in host cells that can damage the virus genome which ROS enhance the pathogenesis ability of infectious influenza that leads to the thymus specific elevation of the mitochondrial superoxide, which interferes with the normal functioning of T-cells lymphocyte damage in influenza A virus infections [32]. Influenza A virus infection causes a rapid influx of inflammatory cells, resulting in increased ROS production, cytokine expression, and acute lung injury which inhibition of this activity would restore host cytokine homeostasis following avian influenza virus infection [33]. Promyelocytic leukemia zinc finger (PLZF) controls the ROS levels that in turn PLZF governs the inflammatory function of natural killer T (NKT) cells, in which ROS regulate homeostasis and effector function of NKT cells, both of which are regulated by PLZF [34]. As mentioned above, anti-viral vaccine activities of $\mathrm{Zn}^{2+}$ ions for viral prevention, entry, replication, and spreading with pathogenesis process are exhibited in Table 1. Zinc-associated vaccine activity mechanism against viruses is indicated that the vaccine activity of released $\mathrm{Zn}^{2+}$ ions from zinc solutions and ZnO NPs, may be enhanced by zinc(II)-induced $\mathrm{Zn}^{2+}$ coordinated adapted immunity, viral growth regulation, and viral apoptosis and death. 
Table 1: Anti-viral vaccine activity of $\mathrm{Zn}^{2+}$ ions for viral prevention, entry, replications, DNA/RNA virus, and spreading into organs.

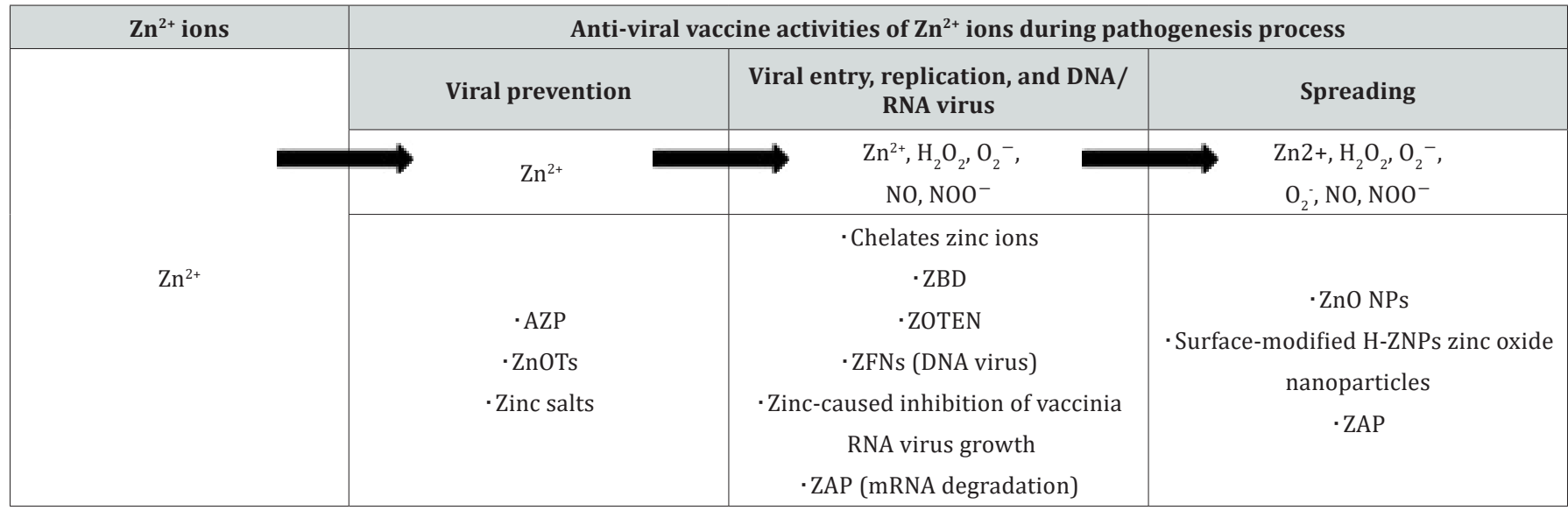

\section{Conclusion}

AZP can efficiently block binding of the viral replication protein, which initiates virus replication, to the replication origin against BSCTV, DNA virus. The AZP phenotypes show strongly resistant to virus infection and viral DNA replication could be applied to the prevention of virus infections in humans. ZnOTs exhibit the ability to neutralize herpes simplex virus type-2 (HSV-2) virions and blocking HSV-2 attachment activity by ZnOTs can have prophylactic as well as therapeutic applications. Zinc salts can mediate antiviral activity on RSV by altering the ability of the cell to support RSV replication. The effect of zinc sulfate on seroconversion after a simple method vaccination had been identified that accelerated HB vaccination can shorten duration of immunization of this clinical trial for showing its effectiveness. The inhibition of zinc binding activity of hMPV M2-1 protein can lead to the development of novel, live attenuated vaccines as well as antiviral drugs for pneumoviruses. The CCHH zinc finger motif provides a critical determinant for virulence in mouse and mutations in the $\mathrm{CCHH}$ motif yield potential vaccine candidates for the development of live species-specific attenuated influenza virus vaccines. Chelates zinc ions also inhibit HIV-1 replication. The LAIVs are attracting attention as an effective strategy due to several advantages over inactivated vaccines. Zinc finger reactive compounds also inactivate retroviruses. ZOTEN promoted the presentation of bound HSV-2 virions to mucosal APCs, enhancing $\mathrm{T}$ cell-mediated and $\mathrm{Ab}$-mediated responses to the infection, and thereby suppressing a reinfection.

ZFNs can specifically and efficiently excise HIV-1 proviral DNA from latently infected human $\mathrm{T}$ cells, the resulting from HIV-1-infected human primary $\mathrm{T}$ cells and latently infected $\mathrm{T}$ cells treated with the inducible ZFNs validated that proviral DNA can be excised. The regulated ZFNs in the presence of HIV-1 Tat may provide a safer and novel genome-editing technology for eradicating HIV-1 proviral DNA from infected host cells. Zinc supplementation in cultured HeLa cells inhibits DNA laddering \& caspase-3 activity, and inhibitory effect on influenza virus induced apoptotic death can be determined at an early stage of the infection by zinc treatment. Further, zinc ions inhibit vaccine virus growth, and are involved in zinc-caused inhibition of vaccinia virus growth. The ZAP inhibits HIV-1 infection by viral mRNAs degradation that ZAP inhibits HIV-1 by recruiting both the $5^{\prime}$ and $3^{\prime}$ mRNA degradation to specifically promote the degradation of multiply spliced HIV-1 mRNA. Higher levels of dietary $\mathrm{ZnO}$ could not provide enhanced protection against PRRSV vaccine that higher levels of dietary $\mathrm{ZnO}$ have the potential to stimulate or modulate systemic immune responses after vaccination against PRRSV. ZOTEN provides a platform for virus capture and presentation of neutralized virions to mucosal APCs.

ZAP inhibits the spreading against XMRV which ZAP inhibits viral infection. The treatment of viral infections with viral spreading will be achieved and improved the therapeutic effects by using zinc oxide nanoparticles. ROS and RNS within viruses of viral entry, viral replication, and viral spread are generated in all situations. ROS induce several cellular effects, including cell cycle progression, apoptosis, DNA damage, senescence, and neurodegeneration. ROS in virus pathogenesis play an important role in cell signaling and regulate hormone action, growth factors, cytokines, transcription, apoptosis, immunomodulation, and neuromodulation, leading to chronic oxidative stress. Oxidative stress has been occurred in various viral infections. The body antioxidant system becomes weaker as HIV progresses that the imbalances inside and outside the cell influence the cell to undergo a programmed cell death. The antioxidant components lead to an excess storage of $\mathrm{H}_{2} \mathrm{O}_{2}$, which further increases the hydroxyl radicals and lipid peroxide that signal the cell to undergo a programmed cell death. The influenza virus induces ROS production in host cells that can damage the virus genome which ROS enhance the pathogenesis ability of infectious influenza that leads to the thymus-specific elevation of the mitochondrial superoxide, which interferes with the normal functioning of T-cells lymphocyte damage in influenza A virus infections. Zinc-associated vaccine activity mechanism against viruses is indicated that the anti-viral vaccine activity of released $\mathrm{Zn}^{2+}$ ions from zinc solutions and ZnO NPs, may be enhanced by 
$\mathrm{Zn}^{2+}$ ion-induced $\mathrm{Zn}^{2+}$ ions-coordinated adapted immunity, viral growth regulation, and viral apoptosis and death.

\section{References}

1. Mothes W, Sherer NM, Jin J, Zhong P (2010) Virus cell-to-cell transmission. J Virol 84(17): 8360-8368.

2. Plum LM, Rink L, Haase H (2010) The essential toxin: Impact of zinc on human health. Int J Environ Res Public Health 7(4): 1342-1365.

3. Baum MK, Shor-Posner G, Campa A (2000) Zinc status in human immunodeficiency virus infection. J Nutr 130(5): 1421S-1423S.

4. Tellinghuisen TL, Marcotrigiano J, Gorbalenya AE, Rice CM (2004) The NS5A protein of hepatitis C virus is a metalloprotein. J Biol Chem 279(19): 48576-48587.

5. Wang N, Dong Q Li J, Jangra RK, Fan M, et al (2010) Viral induction of the zinc-finger antiviral protein is IRF3-dependent but NF-Bindependent. J Biol Chem 285(9): 6080-6090.

6. Lauring AS, Jones JO, Andino R (2010) Rationalizing the development of live attenuated vaccines. Nat Biotechnol 28(6): 573-579.

7. Jang YH, Seong BL (2012) Principles underlying rational design of live attenuated influenza vaccines. Clin Exp Vaccine Res 1(1): 35-49.

8. Cai H, Zhang Y, Ma Y, Sun J, Liang X, et al. (2015) Zinc binding activity of human metapneumovirus M2-1 protein is indispensable for vira replication and pathogenesis in vivo. J Virol 89(12): 6391-6405.

9. Baron S, Fons M, Albrecht T (1996) Viral Pathogenesis, General Concepts. In: Baron S (Eds), Medical Microbiology. ( $4^{\text {th }}$ Edn). Galveston (TX) Chapter 45: 1-7.

10. Sera T (2005) Inhibition of virus DNA replication by artificial zinc finger proteins. J Virol 79(4): 2614-2619.

11. Antoine TE, Mishra YK, Trigilio J, Tiwari V, Adelung R, et al. (2012) Prophylactic, therapeutic and neutralizing effects of zinc oxide tetrapod structures against HSV type-2 infection. Antiviral Res 96(3): 363-375.

12. Suara RO, Crowe JE (2004) Effect of zinc salts on respiratory syncytial virus replication. Antimicrob Agents Chemother 48(3): 783-790.

13. Kaushik N, Subramani C, Anang S, Muthumohan R, Shalimar, et al (2017) Zinc salts block HEV replication by inhibiting the activity of viral RNA dependent RNA polymerase. J of Virology 91(21): 1-13.

14. Afsharian M, Vaziri S, Janbakhsh AR, Sayad B, Mansouri F, et al. (2011) The effect of zinc sulfate on immunologic response to recombinant hepatitis B vaccine in elderly. Hepat Mon 11(1): 32-35.

15. Hui EK, Smee DF, Wong MH, Nayak DP (2006) Mutations in influenza virus $\mathrm{M} 1 \mathrm{CCHH}$, the putative zinc finger motif, cause attenuation in mice and protect mice against lethal influenza virus infection J Virol 80(12): 5697-5707.

16. Pannecouque C, Szafarowicz B, Volkova N, Bakulev V, Dehaen W, et al. (2010) Inhibition of HIV-1 replication by a bis-thiadiazolbenzene1,2diamine that chelates zinc ions from retroviral nucleocapsid zinc fingers. Antimicrob Agents Chemother 54(4): 1461-1468.

17. Briknarová K, Thomas CJ, York J, Nunberg JH (2011) Structure of a zinc-binding domain in the Junin virus envelope glycoprotein. J Biol Chem 286(2): 1528-1536.

18. Jang YH, Seong BL (2012) Principles underlying rational design of live attenuated influenza vaccines. Clin Exp Vaccine Res 1(1): 35-49.
19. Boukhvalova MS, Prince GA, Blanco JC (2010) Inactivation of respiratory syncytial virus by zinc finger reactive compounds. Virol J $7: 1-10$.

20. Antoine TE, Hadigal SR, Yakoub AM, Mishra YK, Bhattacharya P, et al. (2016) Intravaginal zinc oxide tetrapod nanoparticles as novel immunoprotective agents against genital herpes. J Immunol 196(11): 4566-4575.

21. Shi W, Bu P, Liu J, Polack A, Fisher S, et al. (1999) Human papillomavirus type 16 E7 DNA vaccine: Mutation in the open reading frame of E7 enhances specific cytotoxic T-lymphocyte induction and antitumor activity. J Virol 73(9): 7877-7881.

22. Ji H, Lu P, Liu B, Qu X, Wang Y, Jiang Z, et al. (2018) Zinc-finger nucleases induced by HIV-1 tat excise HIV-1 from the host genome in infected and latently infected cells. Mol Ther Nucleic Acids 12: 67-74.

23. Liang C, Wainberg MA, Das AT, Berkhout B (2016) CRISPR/Cas9: a double-edged sword when used to combat HIV infection. Retrovirology 13(1): 37-41.

24. Misaki Wayengera (2011) Identity of zinc finger nucleases with specificity to herpes simplex virus type 0 genomic DNA:novel HSV-2 vaccine/therapy precursors. Theor Biol Med Model 8: 1-13.

25. Srivastava V, Rawall S, Vijayan VK, Khanna M (2009) Influenza a virus induced apoptosis: Inhibition of DNA laddering \& caspase-3 activity by zinc supplementation in cultured Hela cells. Indian J Med Res 129(5): 579-586.

26. Zaslavsky V (1979) Inhibition of vaccinia virus growth by zinc ions: Effect on early RNA and Thymidine kinase synthesis. Journal of Virology 29(1): 405-408.

27. Zhu Y, Chen G, Lv F, Wang X, Ji X, et al. (2011) Zinc-finger antiviral protein inhibits HIV-1 infection by selectively targeting multiply spliced viral mRNAs for degradation. Proc Natl Acad Sci U S A 108(38): 15834-15839.

28. Chai W, Wang Z, Janczyk P, Twardziok S, et al. (2014): Elevated dietary zinc oxide levels do not have a substantial effect on porcine reproductive and respiratory syndrome virus (PRRSV) vaccination and infection. Virol J 11: 1-140.

29. Antoine TE, Hadigal SR, Yakoub AM, Mishra YK, Bhattacharya P, et al. (2016) Intravaginal zinc oxide tetrapod nanoparticles as novel immunoprotective agents against genital herpes. J Immunol 196(11): $4566-4575$

30. Wang X, Tu F, Zhu Y, Gao G (2012) Zincfinger antiviral protein inhibits XMRV infection. PLoS One 7(6): e39159.

31. Singh L, Kruger HG, Maguire GEM, Govender T, Parboosing R (2017) The role of nanotechnology in the treatment of viral infections. Ther Adv Infect Dis 4(4): 105-131.

32. Reshi ML, Su YC, Hong JR (2014) RNA viruses: ROS-Mediated Cell Death. Int J Cell Biol 1-16.

33. Ye S, Lowther S, Stambas J (2015) Inhibition of reactive oxygen species production ameliorates inflammation induced by influenza A viruses via upregulation of SOCS1 and SOCS3. J Virol 89(5): 2672-2626.

34. Kim YH, Kumar A, Chang CH1, Pyaram K (2017) Reactive oxygen species regulate the inflammatory function of NKT cells through promyelocytic leukemia zinc finger. J Immunol 199(10): 3478-3487. 
This work is licensed under Creative Commons Attribution 4.0 License

DOI: 10.19080/CTBEB.2019.19.556012

\section{Your next submission with Juniper Publishers} will reach you the below assets

- Quality Editorial service

- Swift Peer Review

- Reprints availability

- E-prints Service

- Manuscript Podcast for convenient understanding

- Global attainment for your research

- Manuscript accessibility in different formats (Pdf, E-pub, Full Text, Audio)

- Unceasing customer service

Track the below URL for one-step submission https://juniperpublishers.com/online-submission.php 